\title{
Redes de Atenção à Saúde: a percepção dos médicos trabalhando em serviços de urgência
}

\author{
Health Care Networks: the perception of physicians working in \\ emergency services
}

Diana Pereira Lima', Maísa Tavares de Souza Leite ${ }^{2}$, Antônio Prates Caldeira $\mathbf{3}$

RESUMO Objetivou-se conhecer a percepção de médicos em serviços de urgência sobre as Redes de Atenção à Saúde. Trata-se de pesquisa qualitativa, com referencial fenomenológico. O estudo foi realizado em três hospitais de uma capital brasileira. Os participantes foram médicos dos hospitais e plantonistas do Serviço de Atendimento Móvel de Urgência (Samu). Destacaram-se as dimensões: particularidades do médico da urgência, (des)integração e constituição das redes, movimentos do paciente na rede assistencial e interposição no ato profissional. As percepções suscitam a necessidade de intervenções que alinhem conceitos inerentes à organização em redes da atenção à concepção vivida na prática.

PALAVRAS-CHAVE Sistemas de saúde; Pessoal de saúde; Serviços médicos de emergência; Pesquisa qualitativa.

ABSTRACT It was aimed to know the perception of physicians in emergency departments on health care networks. This is a qualitative study, with phenomenological reference. The study was conducted in three hospitals of a Brazilian capital. Participants were doctors from the hospitals and physicians on duty of the Mobile Emergency Care Service (Samu). Stood out the dimensions: particularities of emergency physicians, (dis)integration and constitution of networks, movements of patients into the health care network and interposition in the professional act. The perceptions raise the need for interventions that align concepts inherent to the organization in health care networks to the conception experienced in practice.

KEYWORDS Health systems; Health personnel; Emergency medical services; Qualitative research.

1 Fundação Hospitalar do Estado de Minas Gerais (FHEMIG) - Belo Horizonte (MG), Brasil. dillim2004@yahoo.com.br

2 Universidade Estadual de Montes Claros (Unimontes) - Montes Claros (MG), Brasil. maisa.leite@unimontes.br

3 Universidade Estadual de Montes Claros (Unimontes) - Montes Claros (MG), Brasil.

antonio.caldeira@unimontes.br 


\section{Introdução}

A assistência à saúde, no Brasil, vem passando por importantes transformações desde a promulgação da Constituição Federal de 1988, que criou as condições para instituir-se o Sistema Único de Saúde (SUS). A consolidação de um novo modelo assistencial se constrói paulatinamente no aspecto constitucional, relativo à conquista de direitos individuais e coletivos. Pela legitimidade dessa conquista, são válidas práticas destinadas a evitar, minorar e eliminar elementos considerados, por razões sociais ou técnicas, como problemas de saúde, com adoção de modelos de atenção e bons métodos de gestão (SILVA, 2009). O modelo de atenção à saúde, preconizado pelo SUS, tem ênfase na Estratégia Saúde da Família (ESF) e nos princípios da Atenção Primária à Saúde (APS). Essa proposta destaca a atuação profissional com vínculo e responsabilização pelo usuário e deve desenvolver esforços para melhorar a coerência e a coordenação na prestação de serviços em Redes de Atenção à Saúde (RAS) (SALTMAN; RICO; BOERMA, 2010).

A noção de sistemas e serviços de saúde organizados em redes assistenciais tem sido bastante discutida. Redes são entendidas como estruturas nas quais um conjunto de pontos de intercessão está ligado por um conjunto de relacionamentos em processo dinâmico; implicam pessoas e organizações com autonomia e governança singular, interligadas por tecnologias de informação e comunicação. Assim, essas redes devem ser ativas e fundamentadas em relacionamentos (permuta de ações) entre unidades de prestação de serviços de saúde. A principal finalidade da logística de serviços em redes é dinamizar as instituições no enfrentamento da complexidade em ambientes organizacionais (MENDES, 2011; SILVA, 2011).

É fácil compreender que os serviços de APS sejam mais inteirados com base na ideia de organização em rede. Ainda assim, nem sempre os gestores de saúde têm propiciado as garantias e condições necessárias para assegurar a efetiva atuação na rede assistencial (GONÇALVES ET AL., 2014). Devem-se considerar, ainda, as singularidades e peculiaridades de outros serviços de saúde, cuja produção entrelaça profissionais e usuários com distintas percepções sobre o modelo de atenção e a logística em redes. Cabe destacar que a saúde, como elemento fundamental para o debate sobre constituição e estruturação da atenção em redes, tem primazia no mundo da vida, e que os usuários do sistema, independentemente da complexidade de seus problemas, esperam uma resposta efetiva dos profissionais de saúde. Tal expectativa traz em si a ideia de um campo social onde se articulam forças em constante negociação (CECílıO, 2009).

É nesse contexto que se torna pertinente discutir a percepção do modelo e das redes assistenciais por diferentes atores. O trabalho em saúde é atividade que resulta da influência paradigmática da ciência sobre o processo saúde-doença. É um trabalho vivo em ato, isto é, produz um serviço e o consome no ato de sua produção. Essa particularidade caracteriza o ato em saúde como um produto a ser consumido, cujo valor de uso tem a finalidade perseguida pelo usuário (MERHY ET AL., 2004). É no campo do trabalho que protagonistas da produção em saúde agem, segundo sua ótica e sua percepção, nas cenas que representam o modelo ideal de redes regionalizado e hierarquizado. Esse agir é, necessariamente, atravessado em inter-relações tensionadas, aparatos tecnológicos e um fazer imbuído de valores, crenças, hábitos e trajetórias de vida (MERHY ET AL., 2004; FRANCO, 2006).

Nesse sentido, redes não se formam pelo simples ajuntamento de serviços ou organizações, e a concepção de redes tem influência sobre o fazer em saúde. As concepções profissionais, de certa forma, definem os sentidos das ações que podem modificar as fronteiras institucionais e as práticas da assistência. Nesse cenário, os médicos, pela participação intelectual ativa no ambiente organizacional em que se desenvolve o 
processo de cuidado, desempenham um importante papel no processo de construção efetiva das redes assistenciais, aspecto que tem sido destacado em outros países (GIOVANELLA, 2014). Tal consideração trouxe à tona a necessidade de se conhecer o que pensam os médicos que estão em posição oposta àqueles da APS, em unidades de atendimento à urgência e emergência, onde a restauração do estado de saúde e/ou da própria vida é essencial. A pergunta que norteou este estudo foi: Como os médicos dos serviços de urgência percebem a RAS? Em muitas ocasiões, esses profissionais vivenciam as falhas de vários pontos da rede assistencial quando assistem pacientes vítimas de condições que poderiam ser evitadas a partir de intervenções adequadas e oportunas. Ademais, é também para outras instâncias que esses profissionais devem referenciar os pacientes assistidos e que necessitem de continuidade dos cuidados. Definindo-se a necessidade de conhecer mais profundamente a percepção dos profissionais que dão vida à rede em seus diversos pontos de atenção, o presente estudo buscou compreender a percepção de RAS segundo os médicos que atuam em serviços de urgência de uma capital brasileira.

\section{Método}

Trata-se de pesquisa com abordagem qualitativa, pautada no referencial fenomenológico e em análise na modalidade estrutura do fenômeno situado (POPE; MAYS, 2009; MARTINS; BICUDO, 2005). Entende-se que a pesquisa qualitativa produz, em ambiente natural - no qual o pesquisador participa de forma direta e prolongada -, resultados expressos em descrições de acordo com o fenômeno contextualizado (MARTINS; BICUDO, 2005). De forma mais direta, o uso da abordagem qualitativa (e do método fenomenológico) se destina a questões para as quais, antes de quantificar, é necessário desvelar a compreensão subjetiva das pessoas a respeito do fenômeno em estudo (POPE; MAYS, 2009; MARTINS; BICUDO, 2005; OLIVEIRA; LOPES; DINIZ, 2008). Assim, no contato direto com o que está sendo vivido, da forma mais natural e espontânea possível, buscou-se a percepção a respeito do que se está experienciando, uma vez que por meio da descrição da percepção se desvela o fenômeno (OLIVEIRA; LOPES; DINIZ, 2008). Significados complexos podem ser representados por palavras ou expressões isoladas, e, ao proceder à análise, postas em conjunto, são possíveis as operações combinatórias e transformativas da abstração (MARTINS; BICUDO, 2005; OLIVEIRA; LOPES; DINIZ, 2008).

A estrutura desta pesquisa foi desenvolvida fundamentando-se na interação entre ambiente e cena e empregando-se uma entrevista semiestruturada, caracterizada pela flexibilidade. Foi possível, assim, um contato entre entrevistador e entrevistado e a exploração, em profundidade, de aspectos considerados relevantes para o estudo (POPE; MAYS, 2009), sem preocupação ou menção às relações de poder inerentes às formas de organizar os serviços.

De modo geral, em estudo de natureza fenomenológica, a amostragem é intencional, a seleção dos participantes visa indivíduos dispostos a compartilhar suas experiências, pois o propósito é descrever e compreender um determinado fenômeno pela perspectiva daqueles que o vivenciam. Nessa expectativa, no presente estudo, foram definidos como critérios de inclusão: médicos, plantonistas em serviço de urgência, presentes no ambiente e envolvidos na cena do cuidar, dispostos a responder à entrevista. A interrupção da coleta de dados em cena, com captura da estrutura do fenômeno pelos depoimentos de quem o vive, deu-se pela repetição dos mesmos profissionais no ambiente e em cena. As entrevistas foram realizadas entre os meses de junho e agosto de 2011.

O ambiente da pesquisa foi a 'porta de entrada' de serviços de urgência hospitalar, em unidades de urgência de três hospitais públicos estaduais da capital mineira. Foram 
envolvidos tanto os médicos plantonistas dessas unidades como aqueles que chegavam tripulando a ambulância do Serviço de Atendimento Móvel de Urgência (Samu). Nesse ambiente, a cena em que os médicos se envolviam era do movimento contínuo do paciente atendido previamente em outros serviços de saúde da rede, oriundo de seu domicílio, de via pública ou transportado em ambulância do Samu.

Considerando que, segundo Martins e Bicudo (2005), a entrevista fundamentada na metodologia fenomenológica capta os depoimentos mediante o vivido, os médicos foram abordados durante as atividades de trabalho. Vivendo a cena, o profissional comunica-se por meio de sua linguagem, assim como expressa seu pensar, sentir e agir. Cabe ressaltar que abordar o médico, no ambiente descrito e em cena, é mais que um simples convite à participação na coleta de dados; implica ato relacional e requer sintonia entre quem questiona e aquele que responde; pressupõe o estar junto com, numa relação de empatia. A aproximação entre pesquisador e pesquisado, isto é, o ato relacional, começa pelo estar presente no ambiente, em cena, no momento vivido na 'porta de entrada' do serviço de urgência.

Neste estudo, a análise dos depoimentos seguiu as etapas preconizadas por Martins e Bicudo (2005): análise idiográfica (individual) e análise nomotética (análise geral). $\mathrm{O}$ pesquisador assume uma disponibilidade para ouvir as descrições dos participantes, com escuta e leitura livre (flutuante). A escuta de um a um dos relatos possibilitou um processo pormenorizado de perceber, pelo suporte auditivo e por elementos de entonação e prosódia, o sentido do todo em cada depoimento. Definem-se, então, os elementos essenciais de cada relato, transcritos em expressões originárias da fala - unidade de significado (OLIVEIRA; LOPES; DINIZ, 2008). Essas unidades foram agrupadas, e novas reflexões buscaram uma compreensão em profundidade. Inclui-se nessa fase da análise o grau de frequência, convergências e divergências no conjunto de unidades de significado.

Os aspectos éticos foram respeitados na condução do trabalho, assegurando-se o sigilo e a confidencialidade. O profissional disposto a falar preencheu e assinou do Termo de Consentimento Livre e Esclarecido. O protocolo da pesquisa teve aprovação do Comitê de Ética em Pesquisa da Fundação Hospitalar do Estado de Minas Gerais (Parecer n. ${ }^{\circ}$ 006B/2011) e do Comitê de Ética em Pesquisa da Universidade Estadual de Montes Claros (Parecer n. ${ }^{\circ}$ 006/2011).

\section{Resultados e discussão}

O desenvolvimento deste estudo possibilitou compreender significados de RAS em um conjunto de dimensões e temas. Estiveram em cena 49 médicos. Todos responderam ao questionário de caracterização profissional, e 14 responderam a todas as questões da entrevista, permitindo a exposição do fenômeno. Esse número foi decorrente da saturação observada na análise das entrevistas. Antes de apresentar a percepção apreendida a partir das falas dos médicos situados em serviço de urgência, é prudente apresentar as características desses profissionais. Ao estabelecer as particularidades do profissional, também se apresentam considerações sobre o processo de formação médica e habilitação profissional, aspectos que, seguramente, interferem nas percepções pesquisadas.

\section{Particularidades dos médicos na rede de urgência}

As peculiares dos profissionais entrevistados permitem identificar indivíduos graduados há mais de 48 meses, a maioria com experiência em serviços de urgência de 36 meses ou mais, referindo formação em mais de uma especialidade. As principais especialidades citadas incluíam, em ordem de frequência, cirurgia geral, pediatria, clínica 
geral, cirurgia vascular, cirurgia pediátrica e terapia intensiva. Notadamente, não se identificou entre os médicos participantes a formação especializada na área de medicina de urgência, ainda recente no País.

Os médicos entrevistados relataram participação em cursos de capacitação nos últimos seis meses e nomearam, principalmente, os temas: ATLS (Advanced Life Trauma Support) e ACLS (Advanced Cardiac Life Support). Em síntese, os participantes desta pesquisa mostraram interesse em investir em sua qualificação técnica para $o$ atendimento em serviços de urgência. Todavia, observou-se que, entre os assuntos declarados como conteúdo de capacitação, não houve registro de temas relacionados a modelos de atenção, redes assistenciais ou gestão do cuidado em rede.

$\mathrm{Na}$ área de medicina de urgência, a sistematização da informação para a habilitação dos profissionais que atuam em serviços de urgência tem se baseado no padrão anglo-americano e franco-germânico. Corrobora a escolha desse modelo de capacitação a inexistência, entre as residências da área da medicina, de um programa de formação em urgência (NOGUEIRA, 2009). Ressalta-se que o fato da não participação em programas de capacitação com temáticas que envolvem redes de atenção ou gestão de serviços, além da formação orientada por especialidades, pode limitar suas habilidades em lidar com as dimensões organizacionais do sistema de atenção à saúde em redes.

Deve-se registrar que, no Brasil, muitas escolas médicas ainda desenvolvem currículos que fragmentam o conhecimento, estimulando a especialização profissional (ALMEIDA FILHO, 2010). Esse processo tende a afastar o estudante de medicina do contexto de integração de cuidados e sistematização de RAS. Iniciativas para mudanças no ensino da medicina são reportadas por outros autores em pesquisa que analisa a construção de Projeto Pedagógico no modelo tradicional, inovador e avançado (ABDALLA ET AL., 2009). O estudo conclui que as transformações ocorridas são mais evidenciadas nas concepções do que na prática. Nogueira (2009) afirma que a reorientação efetiva da educação médica, com mudanças profundas de concepções de saúde, práticas clínicas e de relações de poder, implica um novo estilo de pensamento com desenvolvimento de habilidades e competências orientadas na realidade organizacional, seja na academia ou no serviço.

Tais observações destacam a necessidade de estruturação de redes na atenção às urgências e, mais amplamente, no sistema de saúde, com estabelecimento de uma institucionalidade, ou seja, com relações vinculadas entre as normativas prescritas de organização em redes e a formação médica. Adicionalmente, os achados convocam para uma discussão sobre a inclusão do médico na atenção às urgências, com definição explícita de regras e acordos acerca da carreira profissional do especialista em medicina de urgência (PAZIN FILHO; SCARPELINI, 2010). A proposição de formação do especialista em medicina de urgência, nesse sentido, deve incluir a temática das redes de atenção, de modo a facilitar a estruturação das ações em rede.

A partir da análise das entrevistas, foram definidas as seguintes dimensões: (a) (Des) integração e constituição na assistência em rede; (b) O movimento do paciente na rede assistencial; e (c) Interposição no ato profissional. A seguir, cada uma dessas dimensões será abordada, conciliando o debate de conteúdos implícitos em cada uma com a literatura específica.

\section{(Des)integração e constituição na assistência em rede}

O primeiro aspecto apontado pelos entrevistados destaca a (des)integração da rede:

[...] vejo a rede de atenção como uma rede fragmentada. Ela tem diversos pontos em que a pactuação é falha. Ela caminha até certos pontos e, dali pra frente, não tem evolução... daí eu 
considerar que a rede é fragmentada (MD6). [...] o problema é a articulação entre esses setores, que não tem muito, não. A gente não vê referência e contrarreferência. Na verdade, a gente vê que (a assistência) é muito individualizada. Então, não funciona como rede, não funciona como deveria (MD8).

O sentido da fragmentação mencionada pelos médicos deve ser analisado à luz do sentido da palavra 'integração', que traz consigo a noção de tornar inteiro, completo, e ainda tem referência com palavras como conexão, coerência e relação. Integração, utilizada na área da saúde, especialmente referindo-se às redes assistenciais, significa coordenação e cooperação entre provedores dos serviços assistenciais. Tem como elemento fundamental a governança exercida pelo Estado. Governança, nesse contexto, é entendida como padrão de articulação e cooperação pactuado entre atores sociais e políticos e inclui, ainda, os arranjos institucionais que coordenam e regulam a troca de bens de cuidado. Baseada nessa concepção, a governança do sistema de saúde desempenha um papel fundamental na sustentabilidade da integração quando se refere às redes de atenção. A gestão deve assumir a necessidade de integralidade do cuidado, pressupondo que os níveis de atenção só poderão dar conta das necessidades ampliadas de saúde da população se funcionarem em redes (SALTMAN; RICO; BOERMA, 2010; MENDES, 2011). A percepção dos médicos registra uma rede que não funciona como tal e produz uma assistência desconexa.

Compreende-se que, na concepção dos médicos, há relação entre governança e integração no ambiente em redes, com interdependência entre os serviços e entre profissionais.

[...] Eu percebo é que, hoje, a atenção à urgência necessita de integração. Percebo que precisa haver uma fusão do estado com o município e participação federal também, para conseguir dissolver essa grande demanda (MD3). [...] A gente (médicos da urgência) não tem esse contato, não tem esse histórico das pessoas, porque não tem o contato com a atenção primária [...] (MD8).

Integração e governança direcionam a integralidade do cuidado e, no ambiente em redes, constituem-se de acordo com disponibilidade e uso de tecnologias e sistema logístico eficaz entre os pontos de atenção à saúde. Cabe salientar que, entre os sistemas logísticos das RAS, além da referência e da contrarreferência, destacadas pelos profissionais, estão os sistemas de identificação e acompanhamento dos usuários, as centrais de regulação, os sistemas de registro eletrônico em saúde e de transporte sanitário (MENDES, 2011).

Evidencia-se que a percepção de redes tem relação com alianças estratégicas, troca de informações entre os profissionais e entre as instituições, requisitos que legitimam o ambiente em rede como ambiente de cuidado. Conjetura-se, pela concepção de redes desvelada neste estudo, que a rede assistencial é percebida como fragmentada, com repercussões sobre o paciente: "[...]o paciente fica pouco assistido. Ficamos sem recurso maior para levá-lo para outro local” (MD13). “[...] porque não tem como resolver essa demanda de Atenção Básica aqui na urgência. Você sente que não vai resolver, sente que aquilo não vai se resolver. O paciente vai ficar perdido" (MD10).

Para os médicos dos serviços de urgência, compreende-se a constituição da assistência em redes dependente dos meios diagnósticos complementares, de consultas com especialistas para a suposta cura ou resolução do problema de saúde. Nesse sentido, os profissionais destacam sobre os pontos de urgência:

[...] A gente sente que... nós temos mais recursos. Assim, quando a gente precisa de uma transferência, de um exame mais sofisticado, a medicação... isso aí sempre tenho encontrado... 
A gente sente essa facilidade (MD2). [...] o médico aqui pode usar qualquer recurso: exame clínico, físico, laboratorial, propedêutica de alto custo (M14).

$\mathrm{Na}$ atenção prestada em serviços de urgência impera a lógica da assistência por especialidades médicas no atendimento de usuários vítimas de trauma, pessoas com sintomas agudos ou com doenças crônicas agudizadas não transmissíveis (PAZIN FILHO; SCARPELINI, 2010; MALTA; MERHY, 2010). Esse formato de atenção utiliza como insumo os recursos centrados em exames, medicamentos, interconsulta com especialista e internação hospitalar, com o intuito de fazer surgir a objetividade da doença, pois sem lesão objetiva não há diagnóstico (MALTA; MERHY, 2010).

O médico, ao externar sua concepção de redes, ressalta o serviço de urgência como ambiente que oferta vários recursos: exame clínico, laboratorial e de alto custo, possibilitando uma assistência mais completa. $\mathrm{Na}$ perspectiva desse profissional, a assistência constituída em redes significa praticar o ato profissional conforme sua formação, acreditando que referir o paciente ao especialista, prescrever medicamentos, utilizar um conjunto de técnicas para a elaboração de um diagnóstico sejam as melhores opções para assistir. Nessa perspectiva, incompletas são as redes que, em sua oferta de ações, não dispõem desses recursos de imediato.

Na prática médica ainda impera o conceito de que cada especialidade detém o conhecimento de manejo de condições clínicas que lhe são peculiares no atendimento de urgências (PAZIN FILHO; SCARPELINI, 2010). Nogueira (2009) afirma que a formação médica ainda se encontra estruturada a partir de um modelo tecnocientífico, portanto, requisita um processo de cuidado com disponibilidade de especialistas e de serviços com maior concentração de densidade tecnológica, como exames complementares e procedimentos invasivos.

Cabe considerar que a formação médica, até recentemente, desenvolveu-se em um sistema de saúde que operava para tratar problemas agudos (doenças infecciosas, traumas) e necessidades prementes de pacientes. Nesse modelo, as ações são de natureza mais imediata, voltadas para a minimização dos sintomas (queixa-conduta) (MENDES, 2011).

Mendes (2011) traz a tipologia da atenção às condições agudas e de atenção às condições crônicas, caracterizando os dois tipos e debatendo o tema segundo as novas necessidades da população, que vivencia um novo perfil nosológico. Para esse autor, a organização de sistemas de saúde, com constituição da assistência em redes, só pode ser alcançada quando houver a mudança no estilo de atendimento, ou seja, de atendimento a agudos para o atendimento de crônicos com retornos ampliados, além de tempo de resposta.

Compreende-se, a partir das inferências realizadas, que tanto usuários quanto profissionais serão beneficiados com mudanças na formação médica e com sistemas de saúde constituídos com modelos de atendimento cujas respostas sejam mais ampliadas. Considera-se, ainda, relevante o empoderamento dos atores sociais envolvidos, sem desprestígio para as possibilidades e limitações implícitas no processo do cuidar.

\section{O movimento do paciente na rede assistencial}

A percepção dos médicos destaca o movimento do paciente entre diferentes pontos de assistência e a responsabilidade dos diferentes níveis assistenciais: "[...] os pacientes não sabem que têm que ir primeiro no posto de saúde pra depois vir no serviço de urgência" (MD1).

[...] fluxo existe teoricamente. Ele parece ser bem usual e daria vazão aos problemas, mas, na hora da prática, a gente ainda tem todos os entraves, que acaba acumulando os pacientes. A sensação é que fica todo mundo meio estagnado, sem conseguir chegar ao final; fica todo mundo no meio. (MD5). 
Nesse contexto, os médicos salientam a falta de informação para o paciente como aspecto complicador ou limitante para o funcionamento da rede assistencial: “[...] Às vezes, fica um pouco deficiente na falta de informação para a sociedade para utilização dos seus recursos" (MD4). "[...] com a própria informação do paciente e a exposição, através dos veículos de comunicação, sobre a maneira como a saúde é apresentada à população" (MD7).

Com a percepção de um fluxo inadequado, os médicos destacam, também, conduções inadequadas para os pacientes. Nos serviços de urgência, os médicos são especialistas em reconhecer sinais e sintomas de risco imediatos e em intervir no sentido de manter a vida das pessoas. Essa situação muda na atenção primária, onde o médico generalista defronta-se com uma variedade maior de apresentação das doenças, estando, inclusive, mais preparado para lidar com as dimensões subjetivas do adoecer humano (MALTA; MERHY, 2010; STARFIELD, 2002).

Ao destacarem as relações entre os níveis de atenção, os médicos reconhecem a grande carga de responsabilidade do primeiro nível de assistência:

[...] quanto maior a atenção primária na saúde, menor a necessidade de leitos. Hoje a gente vive uma transição em que atenção primária não cumpriu seu papel e atenção secundária é sobrecarregada por conta disso. (MD12).

[...] porque tudo que acontece na atenção primária vai influenciar a terciária, onde eu estou trabalhando. Acaba ocupando os hospitais, as UPAs, os postos de saúde. Não resolve o problema de ninguém. (MD5).

Efetivamente, os serviços de APS, pelos atributos que os caracterizam, seriam os mais adequados para assumir a responsabilidade de coordenar o percurso terapêutico do usuário. Funcionando como 'porta de entrada', as estratégias de coordenação podem ser estabelecidas a partir de uma linha de comunicação entre os níveis de atenção.

Compreende-se do tema 'relações entre os níveis de atenção' uma pluralidade de agentes autônomos e independentes, que se entrelaçam, influenciando mutuamente os desempenhos uns dos outros. Desvelase a informação como elemento benéfico para o paciente e satisfatório para o médico, principalmente quando a comunicação é sistematizada.

\section{Interposição no ato profissional}

Essa dimensão trouxe os temas de responsabilidade pela assistência e superlotação nos serviços de urgência. A relação do funcionamento da rede sobre a responsabilidade pela assistência foi destacada em expressões como: "[...] muitas vezes não se tem tempo adequado ou hábil pra fazer diagnóstico para melhor conduta e melhor resultado naquilo que se trabalha" (MD7). "[...] me influencia no sentido de eu ser produtivo, de ver o sofrimento do paciente e estar aqui em dias que poderia estar com minha família" (MD3). "[...] vejo outro grande impacto que é o fato de, muitas vezes, você perceber que não está dando a melhor assistência que você pode dar. É a única, mas não é a ideal" (MD6).

Resgata-se das falas dos profissionais o mito do médico solitário, que incorpora os ideais de conhecimento superior, autossacrifício, compaixão, acessibilidade, o julgamento ético (SABA ET AL., 2012). Esse mito persiste, pois representa valores e crenças que têm beneficiado médicos e pacientes, reforçado pelo paradigma biomédico, pelas expectativas de cura e pelo financiamento de cuidados de saúde. O médico solitário mantém a responsabilidade individual e a prestação de contas, fomentando o orgulho no trabalho, o que pode abranger desde a aplicação de decisões até a perspicácia clínica para descobrir o diagnóstico preciso para um paciente complexo. 
O tema superlotação do serviço de urgência encontra-se com outro aspecto influenciador da prática, exposto nas unidades:

[...] a partir do momento que se tem um volume aumentado de pessoas para se atender, quem trabalha na área de saúde pública, que recebe um paciente e, ao mesmo tempo, tem que receber 2 pacientes, 3 pacientes, 4 pacientes, sem suporte e sabendo que outras unidades estão na mesma condição, sem capacidade de receber o paciente. (MD7).

[...] tem que atender a uma demanda maior...

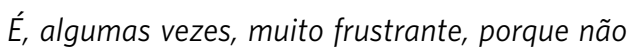
tem como resolver essa demanda de Atenção Básica aqui na urgência. Então, pessoalmente, não é bom. (MD10).

Acerca desse tema, em uma revisão integrativa sobre organização da atenção em urgência, os autores observaram que a insuficiente estruturação da rede de serviços de saúde é o fator que tem contribuído decisivamente para a sobrecarga dos serviços de urgência (AZEVEDo ET AL., 2010). A alta demanda, espontânea ou induzida (regulação do Samu), pelos serviços de urgência gera desorganização da própria unidade, baixa qualidade de atendimento, gastos desnecessários, resultando em uso pouco racional dos recursos disponíveis.

O serviço de urgência responde a uma expectativa da população de forma irregular ou, mesmo, pouco qualificada, pois não compete aos mesmos atuar como responsáveis pelo acompanhamento, seguimento e vínculo com o paciente (PAZIN FILHO; SCARPELINI, 2010; AZEVEDO ET AL., 2010). Na concepção dos médicos, não se consegue trabalhar como se gostaria e ainda responder a demandas que não são, tecnicamente, situações de urgência. A atenção à urgência se estabelece como uma 'porta de entrada' que inclui a urgência não qualificada para tal, o que redunda em efeitos não previstos, principalmente para o usuário portador de doença crônica.

Dentro da temática da superlotação no serviço de urgência, emergem as sensações: pressão, cansaço, constrangimento, frustração, insegurança, expressas nas seguintes declarações: "[...] Trabalho sob pressão o tempo inteiro: pressão da chefia, pressão dos pacientes, da equipe de enfermagem” (MD1). “[...] com o volume aumentado de pacientes, percebo mais cansaço, mais sobrecarregado, exigido" (MD9). "[...] tem que atender a uma demanda maior... Então, pessoalmente, não é bom. Não é só para o paciente, não, para o médico também é muito frustrante, dá uma sensação de incapacidade, de limitação" (MD10).

Refletir sobre o tema superlotação no serviço de urgência conduz o pensamento a duas polaridades: a relação entre RAS, estruturada em vínculos pessoais e modelos de organizações em redes. Há de se considerar a complexidade das relações na produção da saúde, em particular, no atendimento às urgências. Essas relações não se desenvolvem de forma linear, mas se constroem e reconstroem de acordo com os sujeitos envolvidos. A ausência de linearidade imprime subjetividade nas inter-relações estabelecidas em redes e, por conseguinte, exige das organizações flexibilidade institucional para ser mais eficiente. Desse modo, sensações de pressão, cansaço, constrangimento, frustração, insegurança podem moldar as ações dos médicos e também construir e reconstruir, continuamente, interações e trocas instituídas nas RAS.

\section{Considerações finais}

As características identificadas com relação ao perfil dos médicos participantes apontam a necessidade de uma agenda de pesquisa no campo de recursos humanos na área de urgência. É desejável a construção de indicadores e bases de dados e sistemas de seguimento e avaliação que possam delinear o perfil geral de médicos atuantes em serviços de urgência, subsidiando mudanças na graduação e pós-graduação que atendam à atenção em urgências. 
A reflexão compreensiva evidencia a concepção de redes em que o médico se apresenta como sujeito responsável pela assistência. As sensações vividas pelo profissional no serviço de urgência, no contato face a face com o paciente, são decorrentes da crença de que é necessário responder às demandas trazidas com diagnóstico e cura, o que nem sempre é possível. Em outro sentido, a imagem concebida de responsabilidade completa pela assistência gera sensações pouco confortáveis. Esse aspecto pode ter vínculo com a falta de acompanhamento dos pacientes durante todo o percurso terapêutico, ou por não saber como o paciente percorre os caminhos na rede antes e após o evento que deu origem ao atendimento de urgência.

No presente estudo, as proposições de RAS, compreendidas do ponto de vista do médico, tais como integração e constituição da assistência em rede e relações entre os níveis de atenção, sinalizam a necessidade de problematização do tema em um processo de educação permanente.

Ainda sobre a concepção dos médicos acerca das redes assistenciais, registra-se a necessidade premente de construção de um novo estilo de pensamento no atendimento nos serviços de urgência. São desejáveis novos panoramas de prática clínica na produção da atenção à saúde, com estilos permeados pela promoção em saúde, mesmo em presença de situações de urgência. $\mathrm{Na}$ gestão, há de se considerar modelos para além do somatório de ações individuais, oferta de insumos e recursos tecnológicos. Quanto à articulação, é preciso aliar aos elementos técnico-administrativos os saberes dos vários profissionais que atuam nas redes, construindo nova narrativa para o trabalho dos médicos dentro de uma rede de relacionamentos.

Finalizando, é imperativo considerar que as discussões resultantes dos significados da estrutura do fenômeno, discutido aqui à luz da fenomenologia, apresentam aspectos que vão além das normativas. Situam-se no cotidiano, no fazer. Esse fato possibilitou um processo de enumeração, classificação de temas que, em novas pesquisas, responderão a elementos facilitadores da organização da atenção em redes.

\section{Referências}

\footnotetext{
ABDALLA, I. G. et al. Projeto Pedagógico e as Mudanças na Educação Médica. Revista Brasileira de Educação Médica, Rio de Janeiro, v. 33, supl. 1, p. 44-52, 2009.

ALMEIDA FILHO, N. Reconhecer Flexner: inquérito sobre produção de mitos na educação médica no Brasil contemporâneo. Cadernos de Saúde Pública, Rio de Janeiro, v. 26, n. 12, p. 2234-2249, dez. 2010.
}

AZEVEDO, A. L. C. S. et al. Organização de serviços de emergência hospitalar: uma revisão integrativa de pesquisas [internet]. Revista Eletrônica de Enfermagem, v. 12, n. 4, p. 736-745. 2010. Disponível em: <http://www. fen.ufg.br/revista/v12/n4/v12n4a20.htm>. Acesso em: 15 maio 2013.

CECÍLIO, L. C. O. A morte de Ivan Ilitich, de Leon Tolstoí: elementos para pensar as múltiplas dimensões da gestão do cuidado. Interface (Botucatu), Botucatu,v. 13 , supl.1, p. 545-55, 2009.

FRANCO, T. B. As Redes na Micropolítica do Processo de Trabalho em Saúde. In: PINHEIRO, R.; MATTOS, R. A. (Org.). Gestão em Redes: Práticas de avaliação, formação e participação na saúde. Rio de Janeiro: CEPESC-IMS/UERJ-LAPPIS, 2006. 
GIOVANELLA, L. Redes integradas, programas de gestão clínica e generalista coordenador: análise das reformas recentes do setor ambulatorial na Alemanha. Ciência \&t Saúde Coletiva, Rio de Janeiro, v. 16, supl. 1, p. 1081-1096, 2011.

GONÇALVES, C. R. et al. Recursos humanos: fator crítico para as redes de atenção à saúde. Revista Saúde em Debate, Rio de Janeiro, v. 38, n. 100, p. 26-34, jan./ mar. 2014

MALTA, D. C.; MERHY, E. E. O percurso da linha do cuidado sob a perspectiva das doenças crônicas não transmissíveis. Interface (Botucatu), Botucatu, v. 14, n. 34, p. 593-605, 2010.

MARTINS, J.; BICUDO, M. A. V. A Pesquisa Qualitativa em Psicologia: fundamentos e recursos básicos. 5 ed. São Paulo: Centauro. 2005.

MENDES, E. V. As Redes de Atenção a Saúde. 2 ed. Brasília, DF: Organização Pan-Americana da Saúde:Ministério da Saúde, 2011.

MERHY, E. E. et al. (Org.). O Trabalho em saúde: Olhando e experienciando o SUS no cotidiano. 2 ed. São Paulo: HUCITEC. 2004.

NOGUEIRA, M. I. As Mudanças na Educação Médica Brasileira em perspectiva: reflexões sobre a emergência de um novo estilo de pensamento. Revista Brasileira de Educação Médica, Rio de Janeiro, v. 33, n. 2, p. 261-270, abr./jun. 2009.

OLIVEIRA, J. M.; LOPES, R. L. M.; DINIZ, N. M. F. Fenomenologia. Revista Brasileira de Enfermagem, Brasília, DF, v. 61, n. 2, p. 254-257, mar./abr. 2008.
PAZIN FILHO, A.; SCARPELINI, S. Medicina de emergência na FMRP-USP - você pode fugir, mas não há como se esconder! Revista de Escola de Medicina de Ribeirão Preto, Ribeirão Preto, v. 42, n. 4, p. 432-443, 2010.

POPE, C.; MAYS, N. Pesquisa qualitativa na atenção à saúde. 3 ed. Porto Alegre: Artmed. 2009.

SABA, W. G. et al. The myth of the lone physician: toward a collaborative alternative. Annals of Family Medicine, Cleveland, v. 10, n. 2, p. 169-173, 2012.

SALTMAN, R. B.; RICO, A.; BOERMA, W. G. W. (Org.). Atenção primária conduzindo as redes de atenção à saúde: reforma organizacional na atenção primária europeia. Organização Pan-Americana da Saúde. Brasília: Ministério da Saúde, 2010.

SILVA, S. F. Sistema Único de Saúde 20 anos: avanços e dilemas de um processo em construção. Revista Saúde em Debate, Rio de Janeiro, v. 33, n. 81, p. 38-46. 2009.

Organização de redes regionalizadas e integradas de atenção à saúde: desafios do Sistema Único de Saúde (Brasil). Ciência \&t Saúde Coletiva, Rio de Janeiro, v.16, n.6, p. 2753-2762, 2011.

STARFIELD, B. Atenção Primária: equilíbrio entre necessidade de saúde, serviços e tecnologia. Brasília, DF: Ministério da Saúde. 2002.

Recebido para publicação em junho de 2014

Versão final em fevereiro de 2015

Conflito de interesse: inexistente

Suporte financeiro: não houve 